Macedonian Pharmaceutical Bulletin, 66 (Suppl 1) 107 - 108 (2020)

Online ISSN $1857-8969$

UDC: $616.72-002.77-085: 138.178 .8$

DOI: 10.33320/maced.pharm.bull.2020.66.03.053

Short communication

\title{
Development and evaluation of bee venom topical formulation for efficient treatment of arthritis
}

\author{
Lejla Mutapcic ${ }^{1,2}$, Tamara Ivanoska ${ }^{1}$, Angela Mircevska ${ }^{1}$, Eleonora Trajanovska ${ }^{1}$, \\ Ljubica Mihailova $^{1}$, Dushko Shalabalija ${ }^{1}$, Nikola Bijeljanin ${ }^{3}$, Midhat Jasic ${ }^{2}$, \\ Maja S. Crcarevska ${ }^{1}$, Marija Glavas-Dodov ${ }^{1} *$ \\ ${ }^{1}$ Faculty of Pharmacy, UKIM-Skopje, Majka Tereza 47, 1000 Skopje, N. Macedonia \\ ${ }^{2}$ Faculty of Pharmacy, UNTZ, Univerzitetska 8 Tuzla, BiH \\ ${ }^{3}$ Bee cultivator Leshnica, 1000 Skopje, N. Macedonia
}

\section{Introduction}

Arthritis is a chronic, complex autoimmune disease that affects approximately $1 \%$ of the global population. Conventional therapeutic management involves usage of steroids, nonsteroidal antiinflammatory, disease modifying antirheumatic and immunosuppressant drugs. Despite the increasing number of new drugs and treatment regimes, complete long-term disease remission is not achieved for many patients and thus new therapeutic options are required (Guo et al., 2018).

Bee venom (BV) therapy has been used since ancient times. According to animal experiments, BV exhibits antiarthritic, anti-inflammatory and analgesic effects attributable to the suppression of cyclo-oxygenase- 2 and phospholipase A2 expression and a decrease in the levels of TNF- $\alpha$, IL-1 and IL-6, nitric oxide and oxygen-reactive species. Bioactive BV compounds, such as peptides (melittin, adolapin and apamin), enzymes (phospholipase A2) and amines are also associated with these actions (Lee et al., 2014).

The topical delivery is an attractive method for local treatment of inflammatory conditions like musculoskeletal disorders. Topical delivery has many advantages over the conventional oral dosage forms, especially in avoidance of various adverse effects. Having in mind that the therapeutic efficacy of a topical formulation depends on both the nature of the vehicle and the physicochemical properties of the active agent (release rate, rate and extent of drug permeation, etc.) (Özcan et al., 2009), the aim of this study was to develop an effective, stable topical gel formulation containing BV as an active agent.

\section{Materials and methods}

BV was collected by electric stunning, without harming honey bees during July 2019 (Kozarac, $\mathrm{BiH})$ and was stored at $-20{ }^{\circ} \mathrm{C}$. A modified HPLC method (Rybak-Chmielewska and Szczêsna, 2004) was used for assay of BV using melittin (Sigma, USA) as an external standard (Agilent Technologies 1200 Series; Restek Ultra C18 column; gradient elution with $0.1 \%$ trifluoroacetic acid (TFA) in water (mobile phase A) and $0.1 \%$ TFA in acetonitrilewater 80:20 (mobile phase $\mathrm{B}$ ), flow rate $2.5 \mathrm{~mL} / \mathrm{min}$, $20 \mu \mathrm{L}$ injection volume, $\lambda$ of $220 \mathrm{~nm}$ ).

The gels were prepared by dissolving different concentrations of chitosan (CTS, low-molecular weight; Sigma-Aldrich, USA) in $1 \%$ of lactic acid

\footnotetext{
*magl@ff.ukim.edu.mk
} 
solution with or without poloxamer 407 (PL; Pluronic F127, BASF Chemtrade GmbH, Germany) (1.75\% CTS+0.5\% PL-sample 1, 2\% CTS+0.5\% PL-sample 2, $1.75 \%$ CTS-sample 10, 2\% CTS sample 2O, respectively), 5\% of propylene glycol (Alkaloid, N. Macedonia), $0.2 \%$ of potassium sorbate (PS; Apac Chem. Corp., USA) and $0.3 \%$ of $\mathrm{BV}$ by agitatiation at $300 \mathrm{rpm}$ with the aid of magnetic mechanical stirrer (Variomag, Germany).

Prepared gels were characterized for $\mathrm{pH}$ (Sartorius, Germany), viscosity (at $25{ }^{\circ} \mathrm{C}$; DV2T, Spindle T bar T-A 91, Brookfield Eng. Lab., Inc., USA) and spreadability ( $0.5 \mathrm{~g}$ of gel covered with 68 g glass plate, during $5 \mathrm{~min}$ ). PS content was determined UV spectrophotometricaly (264 nm; Lambda 16, Perkin Elmer, USA) after dissolving of $1 \mathrm{~g}$ of gel in $10 \mathrm{~mL}$ of methanol. In vitro $\mathrm{BV}$ release from the gels $(1.5 \mathrm{~g})$ was performed using membrane diffusion cells (MEMBRA-CELL dialysis tubing; Serva Feinbiochemica GmbH, Germany) $\left(32 \pm 0.5^{\circ} \mathrm{C}\right.$, $15 \mathrm{~mL}$ of distilled water as a dissolution medium, $300 \mathrm{rpm})$. At predetermined time intervals aliquots were taken and analyzed by HPLC.

\section{Results and discussion}

BV assay and quality were determined based on melittin content. Obtained BV sample contained $43.54 \%$ of melittin. Prepared gels were characterized with the $\mathrm{pH}$ of 4.5-5.2. Having in mind that the consistency is one of the most important features for analgesic and anti-inflammatory topical forms, the gel viscosity plays an important role in drug permeation control. Results from the viscosity measurements showed that by increasing the CTS concentration, the viscosity of the samples increased (5920 cP for samples $1 O$ and $11160 \mathrm{cP}$ for sample $2 O$, respectively). By incorporation of $0.5 \% \mathrm{PL}$, the viscosity of the gels also increased, probably due to PL properties for micellization and sorting the polymer chains into a denser network (6400 $\mathrm{cP}$ for sample 1 and $18120 \mathrm{cP}$ for sample 2, respectively). All prepared samples showed pseudoplastic behavior. The therapeutic efficacy of gels also depends on their spreadability. The proper spreading helps in the uniform application of the gel to the skin and satisfies the ideal quality for topical application. Spreadability factor calculations demonstrated that sample $2 O$ had the significantly higher spreadability in comparison to sample $1 O \quad(p<0.05)$. By incorporation of PL, both formulations (sample 1 and sample 2) showed similar spreadability as sample 2O. The content of potassium sorbate in formulated gels was $99.85 \pm 2.04 \%$. The concentration of gelling agents (CTS or CTS/PL combinations) significantly influenced the in vitro release behavior of $\mathrm{BV}$ from the prepared formulations. Gel formulation prepared with $1.75 \%$ CTS and $0.5 \%$ PL (sample 1) showed highest release rate $(\sim 98 \%)$ during the period of $24 \mathrm{~h}$, following the Peppas-Sahlin kinetic model.

\section{Conclusion}

According to the results obtained from this study it could be concluded that BV was successfully incorporated into the CTS/PL gels formulations. Prepared gels showed suitable $\mathrm{pH}$ value, viscosity and spreadability. Formulation prepared with $1.75 \%$ CTS and $0.5 \%$ PL could be a promising candidate for efficient topical delivery/treatment of arthritis. Further clinical studies should be conducted.

\section{References}

Guo, Q., Wang, Y., Xu, D., Nossent, J., Pavlos, N.J., Xu, J., 2018. Rheumatoid arthritis: pathological mechanisms and modern pharmacologic therapies. Bone Research 6(15), 1-14. Available at: https://doi.org/10.1038/s41413-018-0016-9.

Lee, J.A., Son, M.J., Choi, J., Jun, J.H., Kim, J., Lee, M.S., 2014. Bee venom acupuncture for rheumatoid arthritis: a systematic review of randomised clinical trials. BMJ Open 4, e006140. Available at: https://doi.org/10.1136/bmjopen-2014-006140.

Özcan, I., Abacı, Ö., Uztan, A.H., Aksu, B., Boyacioğlu, H., Güneri, T., Özer, Ö., 2009. Enhanced topical delivery of terbinafine hydrochloride with chitosan hydrogels. AAPS PharmSciTech. 10(3), 1024-1031. Available at: https://doi.org/10.1208/s12249-0099299-x.

Rybak-Chmielewska, H., Szczêsna, T., 2004. HPLC study of chemical composition of honeybee (Apis mellifera L.) venom. Journal of Apicultural Science 48(2), 103-109.

Maced. Pharm. Bull. 66 (Suppl 1) 107 - 108 (2020) 